\title{
AVALIAÇÃO DA SATISFAÇÃO DOS USUÁRIOS DE APARELHO DE AMPLIFICAÇÃO SONORA INDIVIDUAL - REVISÃO SISTEMÁTICA
}

\section{Assessment of hearing aids users' satisfaction - systematic review}

\author{
Fabiani Figueiredo Magalhães ${ }^{(1)}$, Maria Fernanda Capoani Garcia Mondelli (2)
}

\section{RESUMO}

Tema: satisfação de usuários de aparelho de amplificação sonora individual (AASI). Objetivo: avaliar a o nível de satisfação dos usuários de AASI por meio da aplicação do questionário Satisfaction With Amplification in Daily Life (SADL) e realizar revisão sistemática dos artigos selecionados para o estudo. Conclusão: o $S A D L$ demonstrou ser um instrumento adequado para identificar a satisfação do usuário de AASI, com fácil aplicabilidade e voltado ao uso clínico.

DESCRITORES: Auxiliares de Audição; Questionário; Satisfação

\section{INTRODUÇÃO}

A audição é um dos mais importantes sentidos para o desenvolvimento da comunicação humana ${ }^{1}$.

Um dano qualquer nessa função pode trazer sérias conseqüências ao indivíduo, como a dificuldade de comunicação oral e compreensão adequada das informações sonoras, que, por sua vez, gera uma perda na qualidade de vida².

O Aparelho de Amplificação Sonora Individual (AASI) pode minimizar os problemas causados pela perda da audição, pois permite o resgate da percepção dos sons da fala e dos sons ambientais, promovendo a melhora da habilidade de comunicação ${ }^{3}$.

Durante a orientação aos usuários de AASI existem alguns processos de motivação que devem ser levados em consideração como a aceitação, o benefício e a satisfação ${ }^{4}$. A satisfação é construída de acordo com as impressões subjetivas do indivíduo e, enquanto não ocorrer aceitação, nunca haverá satisfação ${ }^{5}$. Sendo assim, a satisfação é a

(1) Fonoaudióloga; Mestre em Fonoaudiologia pela Faculdade de Odontologia de Bauru - Universidade de São Paulo, FOB/USP.

(2) Fonoaudióloga; Professora Doutora do Departamento de Fonoaudiologia da Faculdade de Odontologia de Bauru Universidade de São Paulo.

Conflito de interesses: inexistente medida do desfecho de reabilitação auditiva que nos mostra o resultado final ${ }^{6}$.

É importante ressaltar, que a variável de maior interesse durante o processo de avaliação da satisfação dos indivíduos com deficiência auditiva é o ponto de vista do paciente e não se relaciona apenas com o desempenho do AASI, e depende exclusivamente das percepções e atitudes do indivíduo ${ }^{7}$. Para que os profissionais conheçam o real benefício ou satisfação do usuário de AASI, assim como também sua eficácia nas diferentes situações do cotidiano, existem questionários que podem fornecer tais informações.

Esses questionários contêm escalas para avaliar o nível de satisfação do indivíduo, pois há vários fatores que influenciam diferentes dimensões relacionadas ao uso do AASI ${ }^{5}$.

O SADL (Satisfaction With Amplification in Daily Life) foi desenvolvido 6 e validado por pesquisadores $^{7-9}$ para aplicação do mesmo na vida diária de usuários de aparelho auditivo para medir o grau de satisfação. $O$ instrumento foi elaborado para avaliar a satisfação de usuários de AASI, quantificandoa por meio de um escore de quatro subescalas: Efeitos Positivos, Custos e Serviços, Fatores Negativos e Imagem Pessoal.

O SADL despontou como um instrumento adequado para estimar a satisfação com o aparelho auditivo por ser sucinto voltado ao uso clínico e permitir a medição objetiva e independente de 
elementos constituintes da satisfação $0^{10,11,12,13,14}$ Portanto, oferece medidas que podem nos nortear a respeito da satisfação do usuário de amplificação sonora e suas dificuldades de comunicação no dia-a-dia ${ }^{15}$.

Considerando que a revisão sistemática é realizada a partir da formulação de perguntas específicas que direcionam a busca das publicações, a pergunta da investigação do presente estudo foi "O questionário $S A D L$ é útil para avaliar a satisfação dos usuários de AASI?" Assim como também o objetivo desse estudo foi verificar o nível de satisfação dos usuários de aparelho de amplificação sonora individual por meio da aplicação do questionário SADL e realizar revisão sistemática de artigos selecionados para o estudo.

\section{MÉTODO}

Seleção dos estudos, critérios de inclusão e extração dos dados.

Para o levantamento dos descritores, utilizou-se o vocabulário estruturado e trilíngue - Descritores em Ciências da Saúde (DeCS), criado pela Bireme para uso na indexação de artigos de revistas científicas, livros, anais de congressos, relatórios técnicos e outros tipos de materiais, assim como, para ser usado na pesquisa e recuperação de assuntos da literatura científica nas bases de dados.

Esse estudo foi realizado por meio de uma pesquisa da literatura médica nas bases de dados da Scielo, Medline e Lilacs, no período de 1996 a 2009. Foram utilizados os termos hearing, hearing aids, satisfaction; job satisfaction, personal satisfaction, questionnaires e effectiveness; amplification, activities daily, patient satisfaction, protocol; evaluation; isoladamente e em combinação.

$\mathrm{Na}$ busca, foram avaliados e selecionados apenas os estudos cujo título, resumo ou corpo do artigo tivessem relação com o objeto do presente estudo e respondessem a questão única genérica: "O questionário SADL é útil para avaliar a satisfação dos usuários de $A A S I$ ?". Após a seleção dos resumos de estudos encontrados, pertinentes à questão proposta, foi realizada a recuperação dos artigos em texto completo.

Os dados de cada um dos artigos potencialmente relevantes para a revisão sistemática foram coletados por meio de uma ficha protocolar contendo: Critérios de elegibilidade do estudo, métodos utilizados, características dos pacientes incluídos no estudo; tipo de intervenções realizadas no estudo, desfechos mensurados e resultados obtidos.

Os artigos selecionados para análise foram os que apresentavam nível de evidência:
1 - revisões sistemáticas e meta análises;

2 - estudos controlados randomizados e

3 - estudos de intervenção não randomizados.

Vale ressaltar que, de acordo com os princípios da revisão sistemática, apenas estudos com níveis de evidência 1 e 2 devem ser selecionados. Contudo, caso este critério fosse adotado, muitas informações relevantes poderiam ser perdidas, visto que na Audiologia poucos estudos apresentam este desenho, revisões sistemáticas e estudos controlados randomizados. Na busca, foram avaliados e selecionados apenas os estudos cujo título, resumo ou corpo do artigo apresentasse relação com o objeto do trabalho. Após a seleção dos resumos de estudos encontrados, pertinentes à questão proposta, foi realizada a recuperação dos artigos em texto completo. A partir dos artigos selecionados foi realizada uma revisão sistemática de acordo com a literatura ${ }^{16}$. Após a leitura do texto completo, os mesmos foram resenhados e classificados de acordo com o tipo de pesquisa, nível de evidência e grau de recomendação.

\section{REVISÃO DA LITERATURA}

Um dos objetivos principais de uma revisão sistemática é agregar fortes evidências de pesquisa para guiar a prática clínica, sendo assim, ao final do levantamento foram encontrados 94 artigos nas bases de dados pesquisadas (tabela 1). No total, apenas 19 enquadraram-se nos critérios de inclusão, sendo classificados quanto aos seus níveis de evidência e grau de recomendação (tabela 2).

A análise dos 19 artigos com relação ao nível de evidência ${ }^{16}$ resultou em 18 (94,73\%) dos artigos classificados como estudo de coorte, 1 (5,26\%) dos artigos classificados como estudo seccionalcruzado. Todos os artigos (100\%) foram classificados com nível de evidência 4 e nota $B$ de recomendação.

Baseado nos artigos selecionados constatouse que o SADL foi apresentado como um instrumento de fácil compreensão e rápida aplicação em $15,78 \%$ dos estudos, sendo observada em $21,05 \%$ uma forte influencia na construção da satisfação, para o efeito positivo do SADL.

Com relação à deficiência auditiva, podemos constatar que é uma das deficiências sensoriais mais devastadoras porque pode causar prejuízos a comunicação e trazer seqüelas emocionais, sociais e ocupacionais ${ }^{17}$.

Dentre as tecnologias e recursos existentes atualmente, os aparelhos de amplificação sonora individual surgiram como um meio de minimizar os efeitos da perda auditiva. 
Tabela 1 - Número de citações encontradas na base de dados Bireme (Medline e Lilacs) de acordo com as estratégias de busca

\begin{tabular}{lcc}
\hline Estratégia de busca - Lilacs & Total & Resposta a questão genérica \\
\hline auxiliares $A N D$ audição $A N D$ questionário & 13 & 1 \\
auxiliares $A N D$ audição $A N D$ satisfação & 12 & 2 \\
avaliação $A N D$ satisfação $A N D$ prótese $A N D$ auditiva & 3 & 0 \\
satisfação $A N D$ prótese AND auditiva & 4 & 0 \\
\hline Estratégia de busca - Medline & Total & Resposta a questão genérica \\
\hline evaluation AND satisfaction AND hearing AND aids & 18 & 0 \\
hearing AND aids AND satisfaction AND protocol & 4 & 0 \\
amplification AND daily life & 30 & 13 \\
hearing AND aids AND satisfaction AND protocol & 4 & 1 \\
personal AND satisfaction AND hearing AND aids & 6 & 2 \\
TOTAL GERAL & $\mathbf{9 4}$ & $\mathbf{1 9}$ \\
\hline
\end{tabular}

Existem muitos fatores que contribuem para o uso bem sucedido da amplificação, como: idade, grau e tipo de perda auditiva, fatores físicos (tamanho da orelha e destreza manual), habilidade de processamento auditivo, uso prévio de AASI e extensão da perda auditiva, que juntos desempenham papel essencial para a aceitação da amplificação. Somado a isso, a percepção do handicap auditivo, custo, expectativas pessoais, satisfação, desempenho e benefício podem indicar, se teremos usuário de aparelho de amplificação sonora satisfeito ${ }^{18}$.

Os 19 artigos selecionados apresentaram sua totalidade de $100 \%$ de satisfação por parte dos pacientes usuários de AASI. Frente a esses resultados, é importante mencionar que monitorar a satisfação é fundamental para avaliar os procedimentos clínicos, garantir os propósitos de qualidade dos serviços e porque a satisfação reflete a realidade dos resultados de saúde. Ao identificar os fatores que contribuem para a satisfação e ao tentar prover tais atributos aos processos envolvidos, tem-se o potencial de obter um resultado mais efetivo nos serviços de saúde ${ }^{19}$.

Alguns estudos indicaram que o efeito positivo tem forte influência na construção da satisfação $0^{6,7,20,21}$. Os autores referiram que essa escala tem importância pelo fato da melhora da comunicação e da qualidade sonora serem identificadas precocemente quando se inicia o uso do AASI e for pouco susceptível a mudanças ao longo do tempo. Porém, um estudo ${ }^{12}$ referiu que os indivíduos que esperam mais benefícios psicológicos e psicoacústicos dos seus aparelhos antes da adaptação tendem a apresentar maior satisfação nestes aspectos após a adaptação. A crença de que o AASI faz a pessoa parecer incompetente é um sério impedimento ao resultado bem sucedido da amplificação e isso é reforçado pela constatação que novos usuários de AASI apresentam expectativa mais baixa que usuários experientes ${ }^{9}$.

Houve menor satisfação na subescala fatores negativos do SADL, especialmente em relação ao uso do telefone em $15,78 \%$ dos estudos.

O presente estudo identificou algumas pesquisas que apontaram a questão 15, sobre o conserto do AASI, como uma limitação do questionário, visto que é difícil de ser avaliada pelos indivíduos adaptados há pouco tempo.

Verificou-se que a aplicação do questionário SADL é mais eficaz após um mês de adaptação ${ }^{20}$, outros estudos sugeriram após 6 semanas $^{12,22}$ e por fim, um que demonstra três meses de adaptação ${ }^{23}$. Com relação a um estudo recente, no qual os pesquisadore ${ }^{24}$ avaliaram a capacidade de usar - AASI em usuários experientes, os resultados sugeriram que os usuários de aparelhos auditivos possuíam experiência e um excelente entendimento de como usar os mesmos.

Foi observado também forte correlação entre os domínios do efeito positivo e serviços e custos, indicando que as queixas sobre as questões acústicas e psicológicas estão associadas à opinião negativa sobre a competência do fonoaudiólogo responsável pela adaptação e sobre a qualidade do AASI.

Outro dado relevante foi com relação à questão dez, sobre a naturalidade do som percebido com o AASI, considerada pelos autores como um dos entraves do SADL. Isso porque é muito difícil de ser avaliada por indivíduos adaptados há muito tempo ${ }^{6}$. Com isto, supõe-se que esses usuários estão acostumados com o som transmitido pelo AASI e não conseguem distinguir diferença dos sons amplificados. 
Tabela 2 - Descrição dos trabalhos analisados segundo nome dos autores, título, ano, nível de evidência grau de recomendação

\begin{tabular}{|c|c|c|c|c|c|}
\hline Autor & Título & Ano & $\begin{array}{l}\text { Nível de } \\
\text { evidencia }\end{array}$ & $\begin{array}{l}\text { Desenho do } \\
\text { Estudo }\end{array}$ & $\begin{array}{c}\text { Grau de } \\
\text { recomendação }\end{array}$ \\
\hline $\begin{array}{l}\text { DESJARDINS, } \\
\text { JL;DOHERTY, KA }\end{array}$ & $\begin{array}{l}\text { Do experienced hearing aind users know } \\
\text { how to use their hearing aids correctly? }\end{array}$ & 2009 & 4 & $\begin{array}{l}\text { Estudo de } \\
\text { coorte }\end{array}$ & B \\
\hline $\begin{array}{l}\text { ROUP, CM; } \\
\text { NOE, CM }\end{array}$ & $\begin{array}{l}\text { Hearing aid outcomes for listeners with high- } \\
\text { frequency hearing loss. }\end{array}$ & 2009 & 4 & $\begin{array}{l}\text { Estudo de } \\
\text { coorte }\end{array}$ & B \\
\hline $\begin{array}{l}\text { MOELLER, MP; } \\
\text { et al }\end{array}$ & $\begin{array}{l}\text { Consistency of hearing aid use in infants } \\
\text { with early-identified hearing loss. }\end{array}$ & 2009 & 4 & $\begin{array}{l}\text { Estudo de } \\
\text { coorte }\end{array}$ & B \\
\hline $\mathrm{OU}, \mathrm{H}$; et al & $\begin{array}{l}\text { Measuring cochlear implant satisfaction in } \\
\text { postlingually deafened adults with the SADL } \\
\text { inventory. }\end{array}$ & 2008 & 4 & $\begin{array}{l}\text { Estudo } \\
\text { seccional- } \\
\text { cruzado }\end{array}$ & B \\
\hline SHI, LF; et al & $\begin{array}{l}\text { Short-term and long-term hearing aid benefit } \\
\text { and user satisfaction: a comparison between } \\
\text { two fitting protocols. }\end{array}$ & 2007 & 4 & $\begin{array}{l}\text { Estudo de } \\
\text { coorte }\end{array}$ & B \\
\hline $\begin{array}{l}\text { TAKAHASHI, G; } \\
\text { et al. }\end{array}$ & $\begin{array}{l}\text { Subjective measures of hearing aid benefit } \\
\text { and satisfaction in the NIDCD/VA follow-up } \\
\text { study. }\end{array}$ & 2007 & 4 & $\begin{array}{l}\text { Estudo de } \\
\text { coorte }\end{array}$ & B \\
\hline $\begin{array}{l}\text { SOARES, DO; } \\
\text { et al. }\end{array}$ & $\begin{array}{l}\text { Satisfação dos usuários de prótese auditiva } \\
\text { em seu dia-a-dia. }\end{array}$ & 2007 & 4 & $\begin{array}{l}\text { Estudo de } \\
\text { coorte }\end{array}$ & $B$ \\
\hline CARVALHO, JSA & $\begin{array}{l}\text { Satisfação de idosos com aparelhos } \\
\text { auditivos concedidos no Estado de } \\
\text { Tocantins. }\end{array}$ & 2007 & 4 & $\begin{array}{l}\text { Estudo de } \\
\text { coorte }\end{array}$ & B \\
\hline $\begin{array}{l}\text { CIENKOWSKI, } \\
\text { KM; et al. }\end{array}$ & $\begin{array}{l}\text { A computer method for assessing } \\
\text { satisfaction with hearing aids. }\end{array}$ & 2006 & 4 & $\begin{array}{l}\text { Estudo de } \\
\text { coorte }\end{array}$ & $B$ \\
\hline $\begin{array}{l}\text { SAUNDERS, GH; } \\
\text { FORSLINE, A }\end{array}$ & $\begin{array}{c}\text { The performance-perceptual test (PPT) and } \\
\text { its relationship to aided reported handicap } \\
\text { and hearing aid satisfaction }\end{array}$ & 2006 & 4 & $\begin{array}{l}\text { Estudo de } \\
\text { coorte }\end{array}$ & B \\
\hline $\begin{array}{l}\text { URIARTE, M; } \\
\text { et al }\end{array}$ & $\begin{array}{l}\text { Measuring hearing aid outcomes using the } \\
\text { Satisfaction with Amplification in Daily Life } \\
\text { (SADL) questionnaire: Australian data. }\end{array}$ & 2005 & 4 & $\begin{array}{l}\text { Estudo de } \\
\text { coorte }\end{array}$ & B \\
\hline $\begin{array}{l}\text { VEIGA, LR; } \\
\text { MERLO, ARC; } \\
\text { MENGUE, SS }\end{array}$ & $\begin{array}{c}\text { Satisfação com a prótese auditiva na vida } \\
\text { diária em usuários do sistema de saúde do } \\
\text { Exército. }\end{array}$ & 2005 & 4 & $\begin{array}{l}\text { Estudo de } \\
\text { coorte }\end{array}$ & B \\
\hline $\begin{array}{l}\text { SAUNDERS, GH; } \\
\text { JUTAI, JW }\end{array}$ & $\begin{array}{l}\text { Hearing specific and generic measures of } \\
\text { the psychosocial impact of hearing aids. }\end{array}$ & 2004 & 4 & $\begin{array}{l}\text { Estudo de } \\
\text { coorte }\end{array}$ & B \\
\hline $\begin{array}{l}\text { HUMES, LE; } \\
\quad \text { et al. }\end{array}$ & $\begin{array}{c}\text { A comparison of two measures of hearing } \\
\text { aid satisfaction in a group of elderly hearing } \\
\text { aid wearers. }\end{array}$ & 2002 & 4 & $\begin{array}{l}\text { Estudo de } \\
\text { coorte }\end{array}$ & B \\
\hline $\begin{array}{c}\text { COX, RM; } \\
\text { ALEXANDER, GC }\end{array}$ & Validation of the SADL questionnaire & 2001 & 4 & $\begin{array}{l}\text { Estudo de } \\
\text { coorte }\end{array}$ & B \\
\hline $\begin{array}{l}\text { HOSFORD-DUNN, } \\
\text { H; HALPERN, J }\end{array}$ & $\begin{array}{l}\text { Clinical application of the SADL scale in } \\
\text { private practice II: predictive validity of fitting } \\
\text { variables. Satisfaction with Amplification in } \\
\text { Daily Life }\end{array}$ & 2001 & 4 & $\begin{array}{l}\text { Estudo de } \\
\text { coorte }\end{array}$ & B \\
\hline $\begin{array}{l}\text { MCLEOD, B; } \\
\text { UPFOLD, L; } \\
\text { BROADBENT, C }\end{array}$ & $\begin{array}{l}\text { An investigation of the applicability of the } \\
\text { inventory, satisfaction with amplification in } \\
\text { daily life, at } 2 \text { weeks post hearing aid fitting }\end{array}$ & 2001 & 4 & $\begin{array}{l}\text { Estudo de } \\
\text { coorte }\end{array}$ & B \\
\hline $\begin{array}{l}\text { HOSFORD-DUNN, } \\
\text { H; HALPERN, J }\end{array}$ & $\begin{array}{c}\text { 1, Clinical application of the satisfaction with } \\
\text { amplification in daily life scale in private } \\
\text { practice I: statistical, content and factorial } \\
\text { validity. }\end{array}$ & 2000 & 4 & $\begin{array}{l}\text { Estudo de } \\
\text { coorte }\end{array}$ & B \\
\hline $\begin{array}{c}\text { COX, RM; } \\
\text { ALEXANDER, GC }\end{array}$ & $\begin{array}{l}\text { Measuring satisfaction with amplification in } \\
\text { daily life: the SADL scale. }\end{array}$ & 1999 & 4 & $\begin{array}{l}\text { Estudo de } \\
\text { coorte }\end{array}$ & B \\
\hline
\end{tabular}


A subescala imagem pessoal foi mantida pelos autores a partir da constatação de que para algumas pessoas a aparência dos aparelhos e a impressão que estes causam aos outros é extremamente significativa, embora vários usuários não se preocupem com tais aspectos ${ }^{6}$.

Os itens da subescala fatores negativos que investigam o desempenho do usuário em ambiente ruidoso; microfonia e uso do telefone foram identificados como insatisfatórios pelos usuários de aparelho auditivo e tal domínio foi, então, criado pelos autores como um termômetro dos problemas da adaptação ${ }^{6}$.

A dificuldade ao uso do telefone foi considerada uma das mais importantes queixas durante a aplicação do SADL verificada pelos autores ${ }^{7,10,11,25}$. Talvez essa queixa ocorra porque falar ao telefone é uma situação auditiva na qual as limitações tecnológicas dos aparelhos auditivos ficam evidenciadas. Por esse motivo, tornam-se necessárias mais orientações com relação ao uso e manuseio do AASI, devendo ser reforçada pelo treinamento de uso do telefone e o aconselhamento por parte dos profissionais responsáveis pelo processo de adaptação. O fonoaudiólogo deve enfatizar que as dificuldades inerentes são inevitáveis, para que o paciente não crie falsas expectativas seguidas de frustração ${ }^{26}$.

Com relação à compreensão de fala no ruído, os autores ${ }^{27}$ informaram que os usuários de AASI de seu estudo apresentaram medidas de satisfação com a prótese auditiva por meio da aplicação do SADL. Relataram ainda, que embora a compreensão da fala no ruído, ou em situações de grupo continua sendo problemática. Os indivíduos relataram usar seus aparelhos auditivos quase todo o tempo em fáceis e difíceis situações de escuta.
O conhecimento proporcionado por esse estudo de revisão sistemática utilizando o SADL será útil para aplicação em diversos indivíduos com perda auditiva, já que esse questionário é importante em várias instâncias, como para verificar a satisfação em mães de crianças usuárias de $\mathrm{AASI}^{26}$, assim como em idosos ${ }^{28}$, em adultos ${ }^{11}$ ou em usuários de implante $\operatorname{coclear}^{29}$ e para determinar o grau de satisfação de acordo com o tipo e modelo do AASI ${ }^{30}$. Além disso, os achados nos indicaram que o SADL pode ser considerado uma avaliação ouro para obter resultados sobre a satisfação de usuários de $A A S{ }^{8}$. A pontuação do SADL é bastante prática e seus índices numéricos permitem entender como o indivíduo se comporta em relação a um grupo normativo e como ele evolui na sua satisfação com o AASI após uma dada intervenção. O instrumento SADL despontou como adequado para estimar a satisfação por ser curto voltado ao uso clínico e por permitir medição objetiva e independentemente de elementos constituintes da satisfação ${ }^{12,31}$.

\section{CONCLUSÃO}

Esse estudo apresentou bom desempenho para identificar a eficácia do questionário após um período de aclimatização com a amplificação com fácil aplicabilidade e voltado ao uso clínico.

As pesquisas demonstraram satisfação dos indivíduos com uso do AASI havendo queixa de maior dificuldade relacionada ao uso do telefone.

O SADL pode ser considerado como avaliação ouro para obter resultados sobre a satisfação do usuário de AASI.

\section{ABSTRACT \\ Background: user satisfaction on hearing aids (HA). Purpose: to ascertain the level of user satisfaction with hearing aids through the application of the questionnaire Satisfaction with Amplification in Daily Life (SADL) and performing a systematic review of articles selected for the study. Conclusion: the SADL proved to be an adequate tool in order to identify user satisfaction with hearing aid, with easy application and returned towards the clinical use.}

KEYWORDS: Hearing Aids; Questionnaire; Satisfaction 


\section{REFERÊNCIAS}

1. Silman S, lório MCM, Mizhahi MM, Parra VM. Próteses auditivas: um estudo sobre seu benefício na qualidade de vida de indivíduos portadores de perda auditiva neurossensorial. Distúrb. Com. 2004;16(2):153-65.

2. Veras RP, Mattos LC. Audiology and Aging: literature review and current horizons. Rev Bras Otorrinolaringol. 2007;73(1):128-34.

3. Ruschel CV, Carvalho CR, Guarinello AC. A eficiência de um programa de reabilitaçãoaudiológica em idosos com presbiacusia e seus familiares. Rev Soc Bras Fonoaudiol. 2007;12(2):95-8.

4. Sandlin RE. Sound Field Assessment: Hearing Aids and Related Issues. In: Sandlin RE. Hearing Aid Amplification: Technical and Clinical Considerations. 2nd ed. San Diego, California: Singular Publishing Group; 2000. p. 343-68.

5. Hosford-Dunn H, Hush JL. Acceptance Benefit and Satisfaction Measures of Hearing Aid User Attitudes. In: Sandlin RE. Hearing Aid Amplification: Technical and Clinical Considerations. 2nd ed. San Diego, California: Singular Publishing Group; 2000: 467-88.

6. Cox RM, Alexander GC. Measuring satisfaction with amplification in daily life: The SADL Scale. Ear and Hearing. 1999;20: 306-20.

7. Hosford-Dunn H, Halpern J. Clinical application of the satisfaction with amplification in daily life scale in private practice I: Statistical, content and factorial validity. J Am Acad Audiol. 2000;11:523-39.

8. Hosford-Dunn H, Halpern J. Clinical application of the SADL scale in private practice II: predictive validity of fitting variables Satisfaction with Amplification in Daily Life. J Am Acad Audiol. 2001;12(1):15-36.

9. Cox RM, Alexander GC. Validation of the SADL Questionnaire. Ear and Hearing. 2001;22:151-60.

10. Veiga LR, Merlo ARC, Mengue SS. Satisfação com a prótese auditiva na vida diária em usuários do sistema de saúde do Exército. Rev Bras Otorrinolaringol. 2005;71(1):67-73.

11. Soares DO, Tavares RA, Ferreira RT, Guglielmino G, Dinato C, Franchi VM. Satisfação dos usuários de prótese auditiva em seu dia-a-dia. Acta ORL/Técnicas em Otorrinolaringologia. 2007;25:290-2.

12. Carvalho JSA. Satisfação de idosos com aparelhos auditivos concedidos no estado do Tocantins. Arquivos Intern de Otorrinolaring 2007;11:462-70.

13. Lessa AH, Costa MJ, Becker KT, Vaucher VA. Satisfação do usuários de próteses auditivas, com perda auditiva de graus severo e profundo. Arq. Int. Otorrinolaringol. 2010;14(3):338-45.
14. Farias RB, Russo ICP. Saúde auditiva: estudo do grau de satisfação de usuários de aparelho de amplificação sonora individual. Rev. soc. bras. fonoaudiol. 2010;15(1): 26-31.

15. Gordo A, Scharlach RC, lório MCM. Avaliação do benefício de próteses auditivas com diferentes tipos de processamento do sinal por meio da aplicação do questionário APHAB. Distúrb com. 2005;17(2):191-202.

16. Cox RM. Evidence-based practice in provision of amplification. J Am Acad Audiol. 2005;16(7):419-38.

17. Russo ICP. Distúrbios da Audição: A Presbiacusia. In: Russo ICP. Intervenção Fonoaudiológica na Terceira Idade. Rio de Janeiro: Ed. Revinter; 1999. p. 51-92.

18. Bongiovani R. Principles of Posfitting Rehabilitation. In: Sandlin RE. Hearing Aid Amplification: Technical and Clinical Considerations. 2nd Edition. San Diego, California: Singular Publishing Group; 2000. p. 439-66.

19. Crow R, Gage H, Hampson S, Hart J, Kimber A, Storey L, Thomas $H$. The measurement of satisfaction with healthcare: implications for a systematic review of the literature. Health Technol Assess. 2002; 6:1-256.

20. Humes LE, Wilson DL, Humes L, Barlow NN, Garner CB. A comparison of two measures of hearing aid satisfaction in a group of elderly hearing aid wearers. Ear Hear. 2002;223(5):422-7.

21. Mcleod B, Upfold L, Broadbent C. An investigation of the applicability of the inventory, satisfaction with amplification in daily life, at 2 weeks post hearing aid fitting. Ear Hear. 2001;22(4):342-7.

22. Saunders GH, Forsline A. The performanceperceptual test (PPT) and its relationship to aided reported handicap and hearing aid satisfaction. Ear Hear. 2006;27(3):229-42.

23. Shi LF, Doherty KA, Kordas TM, Pellegrino JT. Short-term and long-term hearing aid benefit and user satisfaction: a comparison between two fitting protocols. J Am Acad Audiol. 2007;18(6):482-95.

24. Desjardins JL, Doherty KA. Do experienced hearing aind users know how to use their hearing aids correctly? Am J Audiol. 2009;18(1):69-76.

25. Cienkowski KM, McHugh MS, McHugo GJ, Musiek FE, Cox RM, Baird JC. A computer method for assessing satisfaction with hearing aids. Int $\mathrm{J}$ Audiol. 2006;45(7):393-9.

26. Moeller MP, Hoover B, Peterson B, Stelmachowicz P. Consistency of hearing aid use in infants with early-identified hearing loss. Am J Audiol. 2009;18(1):14-23.

27. Takahashi G, Martinez CD, Beamer S, Bridges $\mathrm{J}$, Noffsinger D, Sugiura K, et al. Subjective measures of hearing aid benefit and satisfaction in 
the NIDCD/VA follow-up study. J Am Acad Audiol. 2007;18(4):323-49.

28. Uriarte M, Denzin L, Dunstan A, Sellars J, Hickson L. Measuring hearing aid outcomes using the Satisfaction with Amplification in Daily Life (SADL) questionnaire: Australian data. J Am Acad Audiol. 2005;16(6):383-402.

29. Ou H, Dunn CC, Bentler RA, Zhang X. Measuring cochlear implant satisfaction in postlingually deafened adults with the SADL inventory. J Am Acad Audiol. 2008;19(9):721-34.

30. Roup CM; Noe CM. Hearing aid outcomes for listeners with high-frequency hearing loss. Am J Audiol. 2009;18(1):45-52.

31. Saunders GH, Jutai JW. Hearing specific and generic measures of the psychosocial impact of hearing aids. J Am Acad Audiol. 2004;15(3):238-48.

doi: 10.1590/S1516-18462011005000016

RECEBIDO EM: 01/03/2010

ACEITO EM: 06/11/2010

Endereco para correspondência:

Fabiani Figueiredo Magalhães

Rua Anna Pietro Forte 2-91 - Villaggio I

Bauru - SP

CEP: 17018-820

E-mail: figueiredomagalhaes@uol.com.br 